\title{
Machine Learning Models for Predicting Neonatal Mortality: A Systematic Review
}

\author{
Cheyenne Mangold ${ }^{a} \quad$ Sarah Zoretic ${ }^{a} \quad$ Keerthi Thallapureddy ${ }^{a}$ Axel Moreira ${ }^{b}$ \\ Kevin Chorath ${ }^{c}$ Alvaro Moreira ${ }^{a}$ \\ aDepartment of Pediatrics, University of Texas Health San Antonio, San Antonio, TX, USA; b Department of Pediatrics, \\ Baylor College of Medicine, Houston, TX, USA; 'Department of Otolaryngology, University of Pennsylvania, \\ Philadelphia, PA, USA
}

\section{Keywords}

Artificial intelligence $\cdot$ Neonate $\cdot$ Mortality $\cdot$ Systematic review

\begin{abstract}
Introduction: Approximately 7,000 newborns die every day, accounting for almost half of child deaths under 5 years of age. Deciphering which neonates are at increased risk for mortality can have an important global impact. As such, integrating high computational technology (e.g., artificial intelligence $[\mathrm{Al}]$ ) may help identify the early and potentially modifiable predictors of neonatal mortality. Therefore, the objective of this study was to collate, critically appraise, and analyze neonatal prediction studies that included Al. Methods: A literature search was performed in PubMed, Cochrane, OVID, and Google Scholar. We included studies that used $\mathrm{Al}$ (e.g., machine learning (ML) and deep learning) to formulate prediction models for neonatal death. We excluded small studies ( $n<500$ individuals) and studies using only antenatal factors to predict mortality. Two independent investigators screened all articles for inclusion. The data collection consisted of study design, number of models, features used per model, feature importance, internal and/or external validation, and calibration analysis. Our primary outcome was the average area under the receiving characteristic
\end{abstract}

karger@karger.com

(c) 2021 S. Karger AG, Basel

www.karger.com/neo

Karger" curve (AUC) or sensitivity and specificity for all models included in each study. Results: Of 434 articles, 11 studies were included. The total number of participants was $1.26 \mathrm{M}$ with gestational ages ranging from 22 weeks to term. Number of features ranged from 3 to 66 with timing of prediction as early as 5 min of life to a maximum of 7 days of age. The average number of models per study was 4 , with neural network, random forest, and logistic regression comprising the most used models (58.3\%). Five studies (45.5\%) reported calibration plots and $2(18.2 \%)$ conducted external validation. Eight studies reported results by AUC and 5 studies reported the sensitivity and specificity. The AUC varied from $58.3 \%$ to $97.0 \%$. The mean sensitivities ranged from $63 \%$ to $80 \%$ and specificities from $78 \%$ to $99 \%$. The best overall model was linear discriminant analysis, but it also had a high number of features $(n=17)$. Discussion/Conclusion: ML models can accurately predict death in neonates. This analysis demonstrates the most commonly used predictors and metrics for Al prediction models for neonatal mortality. Future studies should focus on external validation, calibration, as well as deployment of applications that can be readily accessible to health-care providers.

(c) 2021 S. Karger AG, Basel

Cheyenne Mangold and Sarah Zoretic contributed equally as co-first authors. 


\section{Introduction}

Neonatal mortality contributes significantly to childhood death rates. According to the World Health Organization, neonates comprise $47 \%$ of deaths among children $<5$ years of age [1]. Therefore, one of the goals of the USA is to reduce worldwide neonatal death by the year 2030 [2]. Identifying factors leading to neonatal deaths may guide future areas for intervention and could assist clinicians when discussing potential outcomes with families.

Artificial intelligence (AI) is an expanding area of research in the medical field. Specifically, machine learning (ML) is a branch of AI that is capable of performing complex tasks that typically require human intelligence. ML approaches recognize patterns, create algorithms, and adapt according to the new data [3]. Compared to traditional logistic/linear regression techniques, ML methods can unravel interactions that are nonlinear or binary and can yield highly stable predictions in large datasets that are oftentimes challenging to find correlations [4]. Furthermore, there are voluminous number of models from which to choose in ML that allows for an optimal algorithm within the same data (e.g., artificial neural networks, random forest, least discriminant analysis, k-nearest neighbor, and even logistic regression [LR] $[3,5,6]$, refer to Figure 1 for a summary comparing traditional statistical methods compared to ML).

Integration of high computational algorithms into the health-care system has improved patient care, safety, and hospital efficiency. For instance, a recent article used computer modeling to inform which in-patient surgical patients were more likely to be discharged within $24 \mathrm{~h}$ [7]. Another example is the use of $\mathrm{AI}$ in intensive care units (ICUs), to alarm providers which patients are at increased risk for sepsis. Notably, similar algorithms have been used to investigate risk factors for death in neonates.

Our interest was to review neonatal studies that used AI models to identify risk factors for deaths. We chose to study AI as this emerging field can analyze complex relationships and recognize patterns to optimize predictive abilities. Thus, we conducted a systematic review to synthesize and examine the predictive ability of AI-based algorithms for neonatal mortality.

\section{Methods}

This review adhered to the guidelines set by the Preferred Reporting Items for Systematic Reviews and Meta-Analyses statement, refer to online suppl. File 1; for all online suppl. material, see www.karger.com/doi/10.1159/000516891 [8]. Our protocol was registered to PROSPERO, the international prospective register of systematic reviews on February 17, 2021; refer to online suppl. File 2 .

\section{Literature Search}

A literature search was performed using MEDLINE's database PubMed, OVID, and Cochrane through December 16, 2020. Search terms included "neonate," "newborn," "infant," "mortality," "death," "artificial intelligence," "machine learning," and "deep learning." Peer-reviewed articles were included. A filter was placed to limit the studies to humans and children. More details can be viewed in online suppl. File 3. Two investigators (S.Z. and C.M.) screened titles and abstracts from the search results and then independently reviewed full-text articles that met inclusion criteria. A third investigator (A.M.) was utilized to resolve any differences in the article selection (see online suppl. File 4 for more details).

\section{Inclusion and Exclusion Criteria}

In this study, neonatal death was described as death within the first 28 days of life per the definition by the World Health Organization [9]. We included all live-born infants, irrespective of preterm or term gestation. For studies including premature newborns, the outcome of mortality was assessed until they were discharged from the hospital. Studies were eligible if they included AI-based algorithms (e.g., ML, deep learning, neural network, etc.) to predict neonatal death. Prospective and retrospective studies that examined both perinatal factors and neonatal factors were included. Studies were excluded if they used only antenatal factors to predict perinatal death or stillborn. Also, studies were not included if neonatal morality was not the primary outcome of the study or if the term newborns died outside the neonatal period. The studies were excluded that had $<500$ patients due to ML models having less accuracy in smaller datasets $[10,11]$.

\section{Primary End Point and Data Extraction}

The primary end point for this study was mortality during the neonatal period. The data were collected by all investigators and compared for accuracy, with an additional investigator who resolved any differences. The additional investigator was utilized on 3 occasions to help resolve the differences during the extraction of data due to ambiguous reporting in study methodologies. The extracted data included the study design (sample size, type of ML model, exclusion criteria, timing of the data, and handling of missing data), ML data (number of variables examined, number of variables in the final models, the variables used in the models, performance metrics, percent of data used to test, train, or internally validate the model, cross-validation, calibration, hyperparameter tuning, and external validation), and if studies compared models to LR. All models used to predict were included (e.g., studies reporting multiple ML models with different combinations of predictors).

\section{Performance Metrics and Data Analysis}

Numerous metrics are typically used in prediction models formulated via AI. The area under the receiver operating characteristic curve (AUC) is the most widely used test to assess the predictive ability of a model. The AUC is a graphic interpretation that incorporates sensitivity (true positive/true positive + false negative) and specificity (true negative/true negative + false positive). We chose 
Fig. 1. Flow diagram of model development. Side by side comparison of ML and LR. ML, machine learning; LR, logistic regression; PPV, positive predictive value.

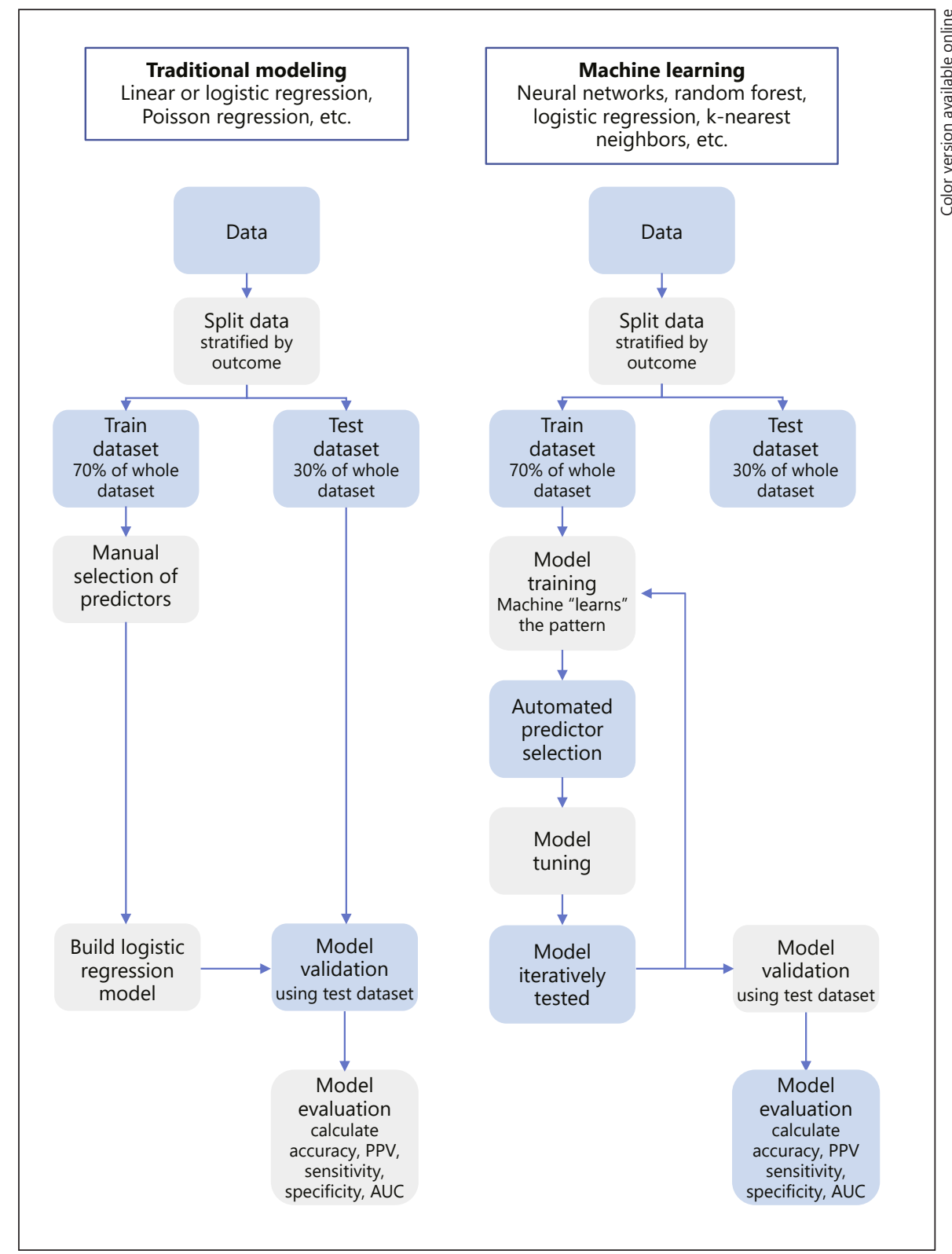

AUC, a priori, as the performance metric. If the study did not report the AUC, we collected the sensitivity and specificity as they are surrogates for the AUC. Since the confidence interval of the AUC was not uniformly reported, we computed the mean with standard deviation (SD) of the AUC for all the prediction models included in each study. This technique has been used in similar systematic reviews $[12,13]$. All analyses and graphics were conducted in $\mathrm{R}$ version 4.0.4.

\section{Risk of Bias}

Risk of bias for each study was evaluated independently by 2 investigators, with a third investigator who acted as a tiebreaker if there were any differences. Risk of bias was assessed with the Checklist for Critical Appraisal and Data Extraction for System- atic Reviews of Prediction Modeling Studies checklist [14]. Level of evidence for the 9 domains was expressed as high, low, or unclear. Reviews of ML studies have identified the underreporting of experimental methods as a source of potential bias as variables can be weighed to heavily or models can be overfitting the data $[15,16]$.

\section{Results}

\section{Study Selection}

After identifying articles through the various databases and checking articles' references for additional studies, 


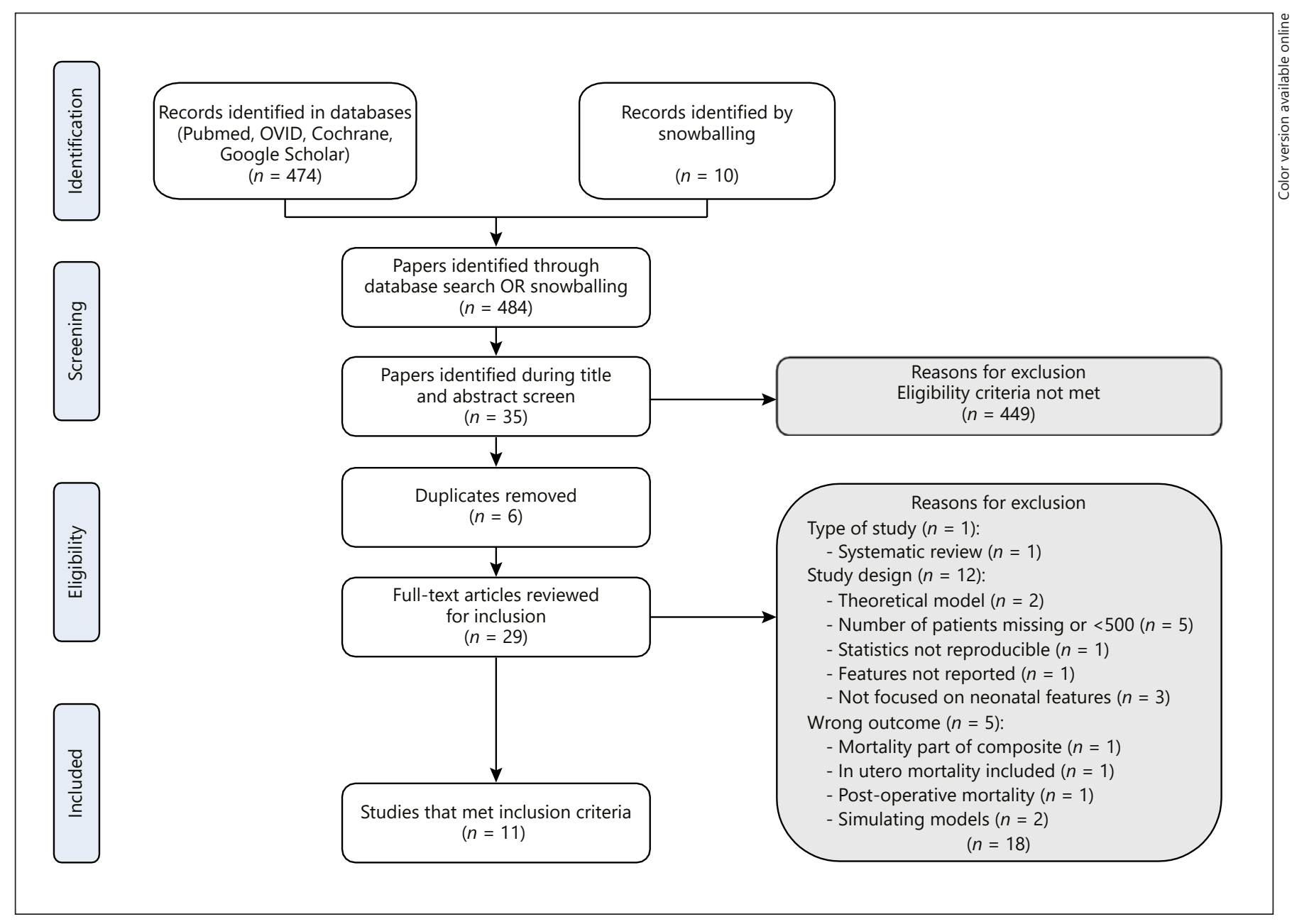

Fig. 2. Flow diagram for study selection.

a total of 484 articles were screened. After screening and removal of duplicates, a total of 29 full-text articles were read and 11 articles met inclusion criteria [17-27]. Reasons for exclusion are described in the flow diagram in Figure 2.

\section{Study Characteristics}

All study populations were taken from various countries, all of which were from North America and Europe. The number of patients in each dataset ranged widely, with a median sample of 850 . The complete study characteristics can be seen in Table 1 .

The studies reported a total of 129 different models, ranging from 1 to 52 models per article. Gilchrist et al. used 2 datasets for their study, one of the sets had $<500$ patients, so the 7 models derived from the small dataset were excluded. The number of features per model ranged from 2 to 66 . Of the 11 studies, 9 (82\%) created $>1$ model. All studies reported the time period over which the data were collected which ranged from collection at delivery through entire hospital stay. A majority of the studies (73\%) reported exclusion criteria for the study.

Handling of missing values was reported in $81.8 \%$ of the articles; the handling of missing data was done by removal of incomplete cases or simple imputation of missing values. Model calibration, hyperparameter tuning, and external validation were done in $<50 \%$ of the articles. Cross-validation was utilized in $73 \%$ of the studies.

\section{Model Design}

The most common ML algorithms were artificial neural networks, LR, and random forest. Traditionally, LR is the most common algorithm for prognostic modeling and therefore often compared to AI-based models [28]. 


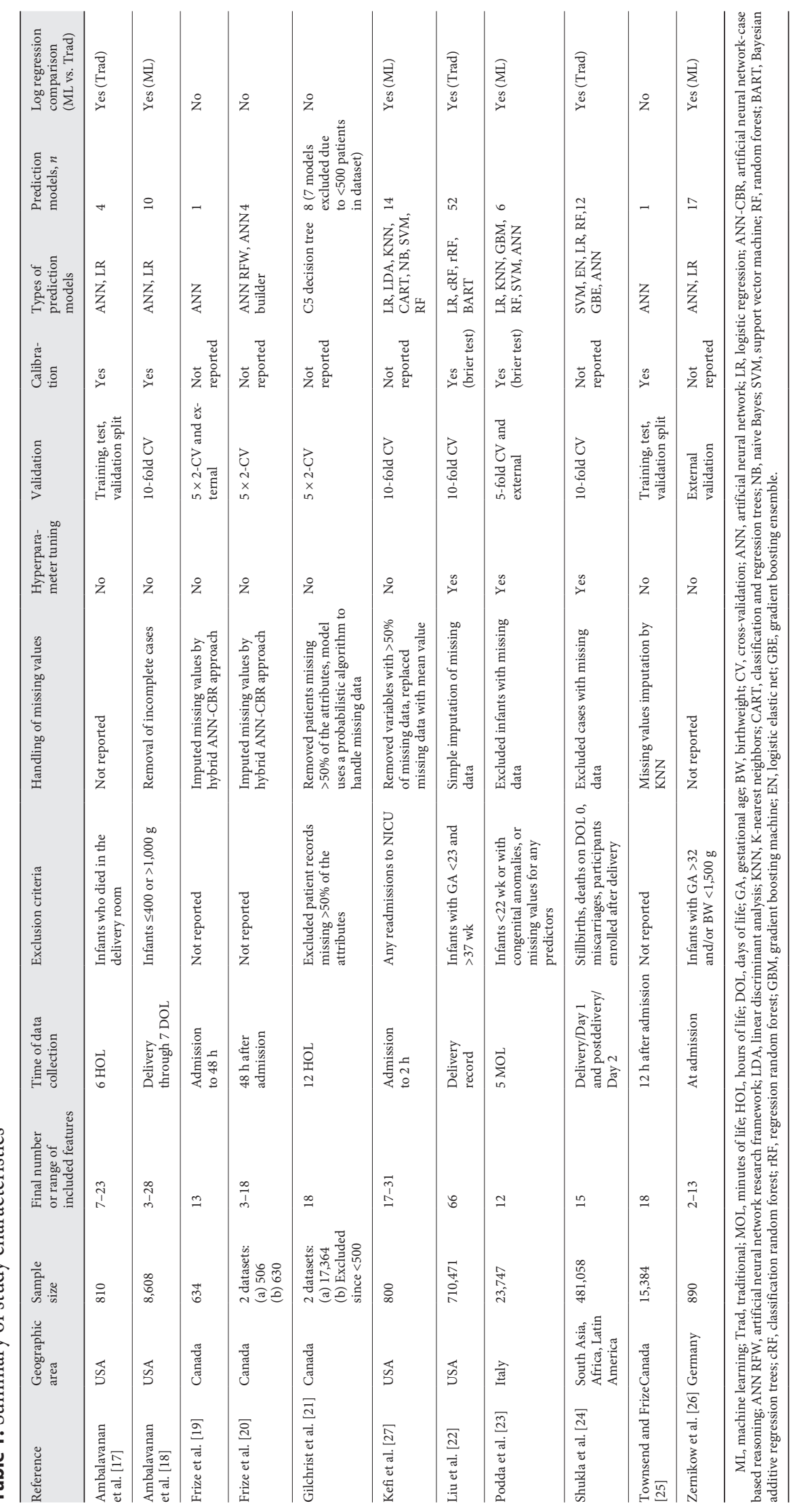


Table 2. Summary of model performance

\begin{tabular}{|c|c|c|c|c|c|c|}
\hline \multirow[t]{4}{*}{ Podda et al. [23] } & LR & 12 & $5 \mathrm{MOL}$ & 0.9098 & NR & NR \\
\hline & $\mathrm{KNN}$ & 12 & $5 \mathrm{MOL}$ & 0.9108 & NR & NR \\
\hline & $\mathrm{RF}$ & 12 & $5 \mathrm{MOL}$ & 0.9138 & NR & NR \\
\hline & GDM & 12 & $5 \mathrm{MOL}$ & 0.9147 & NR & NR \\
\hline \multirow[t]{8}{*}{ Shukla et al. [24] } & SVM & 15 & Delivery/Day 1 & 0.583 & NR & NR \\
\hline & EN & 15 & Delivery/Day 1 & 0.81 & NR & NR \\
\hline & LR & 15 & Delivery/Day 1 & 0.81 & NR & NR \\
\hline & $\mathrm{RF}$ & 15 & Delivery/Day 1 & 0.82 & NR & NR \\
\hline & GBE & 15 & Delivery/Day 1 & 0.825 & NR & NR \\
\hline & RF & 15 & Postdelivery/Day 2 & 0.87 & NR & NR \\
\hline & GBE & 15 & Postdelivery/Day 2 & 0.87 & NR & NR \\
\hline & ANN & 15 & Postdelivery/Day 2 & 0.86 & NR & NR \\
\hline Townsend and Frize [25] & ANN & 18 & $12 \mathrm{HOL}$ & 0.907 & 70.5 & 92.6 \\
\hline \multirow{9}{*}{ Zernikow et al. [26] } & ANN & 13 & At admission & 0.954 & NR & NR \\
\hline & ANN & 12 & At admission & 0.924 & NR & NR \\
\hline & ANN & 12 & At admission & 0.928 & NR & NR \\
\hline & ANN & 12 & At admission & 0.929 & NR & NR \\
\hline & ANN & 12 & At admission & 0.930 & NR & NR \\
\hline & ANN & 12 & At admission & 0.936 & NR & NR \\
\hline & ANN & 12 & At admission & 0.943 & NR & NR \\
\hline & ANN & 2 & At admission & 0.890 & NR & NR \\
\hline & LR & 6 & At admission & 0.917 & NR & NR \\
\hline \multirow[t]{4}{*}{ Ambalavanan et al. [17] } & ANN & 7 & $6 \mathrm{HOL}$ & 0.87 & 80 & 85 \\
\hline & ANN & 23 & $6 \mathrm{HOL}$ & 0.83 & 80 & 69 \\
\hline & LR & 7 & $6 \mathrm{HOL}$ & 0.87 & 80 & 85 \\
\hline & LR & 23 & $6 \mathrm{HOL}$ & 0.82 & 80 & 72 \\
\hline Frize et al. [19] & ANN & 13 & Adm to $48 \mathrm{~h}$ & NR & 63 & 99 \\
\hline \multirow[t]{4}{*}{ Frize et al. [20] } & ANN RFW & 3 & $48 \mathrm{~h}$ after adm & NR & 62 & 99 \\
\hline & ANN RFW & 18 & $48 \mathrm{~h}$ after adm & NR & 68 & 97 \\
\hline & ANN builder & 3 & $48 \mathrm{~h}$ after adm & NR & 72 & 96 \\
\hline & ANN builder & 18 & $48 \mathrm{~h}$ after adm & NR & 81 & 98 \\
\hline Gilchrist et al. [21] & C5 decision tree & 18 & $12 \mathrm{HOL}$ & NR & 56 & 97 \\
\hline
\end{tabular}


Table 2 (continued)

\begin{tabular}{|c|c|c|c|c|c|c|}
\hline \multirow[t]{9}{*}{ Kefi et al. [27] } & LR & 17 & Adm to $2 \mathrm{~h}$ & 0.86 & NR & NR \\
\hline & KNN & 17 & Adm to $2 \mathrm{~h}$ & 0.97 & NR & NR \\
\hline & CART & 17 & Adm to $2 \mathrm{~h}$ & 0.71 & NR & NR \\
\hline & $\mathrm{RF}$ & 17 & Adm to $2 \mathrm{~h}$ & 0.80 & NR & NR \\
\hline & LR & 31 & Adm to $2 \mathrm{~h}$ & 0.91 & NR & NR \\
\hline & LDA & 31 & Adm to $2 \mathrm{~h}$ & 0.97 & NR & NR \\
\hline & KNN & 31 & Adm to $2 \mathrm{~h}$ & 0.57 & NR & NR \\
\hline & CART & 31 & Adm to $2 \mathrm{~h}$ & 0.64 & NR & NR \\
\hline & NB & 31 & Adm to $2 \mathrm{~h}$ & NR & NR & NR \\
\hline \multirow{3}{*}{ Liu et al. [22] } & $\mathrm{rRF}$ & 66 & Delivery record & $0.939 *$ & NR & NR \\
\hline & BART & 66 & Delivery record & $0.940^{*}$ & NR & NR \\
\hline & & 66 & Delivery record & $0.947^{*}$ & NR & NR \\
\hline
\end{tabular}

MOL, minutes of life; HOL, hours of life; DOL, days of life; ANN, artificial neural network; LR, logistic regression; ANN-CBR, artificial neural network-case based reasoning; ANN RFW, artificial neural network research framework; LDA, linear discriminant analysis; KNN, K-nearest neighbors; CART, classification and regression trees; NB, naive Bayes; SVM, support vector machine; RF, random forest; BART, Bayesian additive regression trees; cRF, classification random forest; rRF, regression random forest; GBM, gradient boosting machine; EN, logistic elastic net; GBE, gradient boosting ensemble; NR, not reported; AUC, area under the receiving characteristic curve. *Average c-statistic of all 13 years.

However, this should not be confused with a LR ML approach. Of the studies examined, 4 studies (36\%) compared AI-based models to machine learning LR models $[18,23,26,27]$. Three studies (27\%) compared ML to the traditional LR approach $[17,22,23]$.

\section{Model Performance}

Table 2 shows a summary of model performance. Of the 11 studies, 8 (73\%) reported the AUC. Two articles presented the data with sensitivity, specificity, positive predictive value, and negative predictive value. One article presented the data with only sensitivity and specificity [20]. Most of the prediction models were calculated in the first $48 \mathrm{~h}$ after the neonate was born.

Figure 3 graphically shows the average AUC with SD by study where the boxes show the calculated average AUC, with lines to identify SD and dots to represent AUC outliers. As seen, the mean AUC for the models in the 8 studies that reported AUC were above $70 \%$. Two models had an average AUC $>90 \%[22,26]$. Table 2 reports the sensitivity and specificity for 5 studies. The sensitivity was lower in 9 of the 11 models that included sensitivity and specificity. The model with highest sensitivity and speci- ficity was by Frize et al. [20] which was an artificial neural network with 18 features and a sensitivity and specificity of 81 and $98 \%$, respectively.

In Figure 4, we show the most common predictors accounted for in the studies. The top 4 predictors, also referred to as features, were birthweight, gestational age, Apgar score(s), and multiple births. These predictors were incorporated in at least 7 studies (64\%). The K-nearest neighbor and least discriminant models by Kefi et al. [27], with 17 and 31, respective features, had the highest AUC at $97.0 \%$. The model with the least number of features and highest AUC was a neural network model built by Ambalavanan et al. [18], which had an AUC of $81.6 \%$ and used only 3 variables (prenatal steroids, non-Hispanic blacks, and gestational age).

\section{Risk of Bias}

Currently, there is no widely used tool to assess risk of bias in ML models. For this review, the Checklist for critical Appraisal and data extraction for systematic Reviews of prediction Modeling Studies were used to assess for the risk of bias [14]. Online suppl. File 5 shows the areas where bias was assessed and whether there was high risk, 


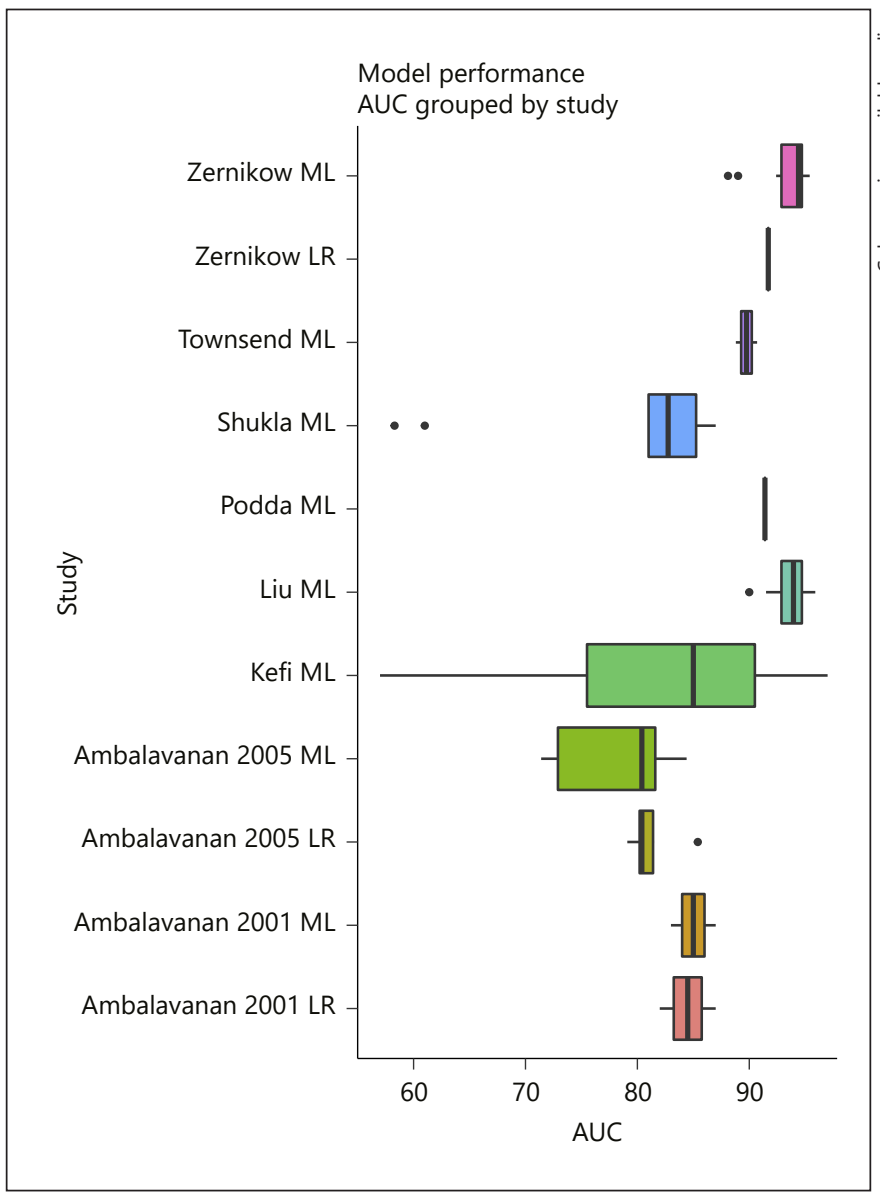

Fig. 3. Average AUC with SDby study. Box and whisker plot displaying average AUC and calculated SD for the 8 studies that reported the AUC. Dots represent outliers. AUC, area under the receiving characteristic curve; SD, standard deviation.

low risk, or unknown risk of bias. All articles had low risk of bias in the areas of data source, outcomes, model performance, and model evaluation. The areas with the most concern for high risk of bias included the handling of missing data and result presentations. The studies where high risk due to limited reporting sample size data, the handling of missing data in the model, and the reporting of the results of the model, and how the results accounted for or adjusted for any missing data.

\section{Discussion/Conclusion}

AI or ML models have paved their way into research because of their ability to recognize complex, nonlinear patterns not otherwise identified by standard methods. There have been many ML models published in adult studies, including those predicting death in a multitude of disease processes [29-33]. To date, there are far fewer AI models published in pediatrics and neonatology. To our knowledge, this is the first study to systematically review and analyze the published ML models predicting death in neonates.

Herein, we conducted a systematic review of 11 studies evaluating the predictive ability of AI models for neonatal mortality. This report included a total of 1.26 million infants with gestational ages ranging from 22 weeks to term. The mean AUC in this review varied from 58.3 to $97.0 \%$ demonstrating their ability to predict mortality in neonates. Despite several advantages of AI for medical prognostication, it is important to recognize the limitations of these techniques. Notwithstanding novel AI models, LR is, in itself, a very powerful tool, even for complex problems, and has been shown to yield comparable predictive performances when used in large population datasets [34]. In this report, the few AI-based algorithms comparing to non-AI-based (e.g., LR) models yielded similar AUC performance.

This review analyzed neonatal prediction models according to the AUC. The AUC, also referred to as C-statistic, is the most common performance measure in describing the discriminative performance of generalized LR/ LR models $[35,36]$. While there is limited research on what specifically defines low versus high discriminative power, Steyerberg et al. [37] claim that models with an AUC around $60 \%$ as having low discriminative power versus high discriminative models having an AUC closer to $95 \%$. Based on these criteria, only 5 of the models in the review had AUCs of $95 \%$ or higher, these were from Kefi et al. [27] and Zernikow et al. [26] with a range of 12-31 features and were models derived from linear discriminant analysis, K-nearest neighbor, and artificial neural network.

Five articles presented the sensitivities and specificities. The mean sensitivities ranged from 63 to $80 \%$ and specificities from 78 to $99 \%$. Tests that are both highly sensitive and specific, generally those $>70 \%$, can indicate clinical utility for the accurate detection of positive and negative results [38]. Of the 11 models that included sensitivity and specificity, only 6 of said models had both sensitivity and specificity above $70 \%$. Those models came from Ambalavanan et al. [17], Frize et al. [20], and Townsend and Frize [25] with a range of 3-18 features, and most were derived from artificial neural networks.

A fair amount of heterogeneity existed among the articles with regard to their validation tools. Only $48 \%$ of studies calibrated their models and an even smaller per- 
Barplot of features included in study models

Birthweight Gestational age APGAR score(s)

Multiple birth

Prenatal steroids Maternal HTN/PIH

Blood pressure

Maternal age

Gravida/Parity

WBC count

Temperature

Respiratory rate

Maternal other $\mathrm{d} / \mathrm{o}$

Hematocrit

Heart rate

Sodium

RDS

Prenatal care

pO2

Platelet count

Placental/uterine disorders

Glucose

Gender

$\mathrm{FiO} 2$

Delivery hemorrhage

Chromosome/congenital anomaly

Chorioamnionitis

Tocolytics

$\mathrm{SaO} 2$

选

Resp support

PROM

Labor issues

pCO2

Oxygenation index/pO2:FiO2

Neutrophil count

Maternal education

Maternal DM/Gestational DM

Income/Insurance status

Inborn/Outborn

Emergency delivery/fetal distress

Delivery mode Cord d/o

BMV resuscitation Antibiotics

Age of neonate

Urine output

SGA

Seizures

PDA meds

Oligohydramnios

Neonatal hospitalization

Maternal antibiotics

Marital status

Labor present

Hospital delivery

Delivery by physicians

Condition on admission

Chest xray

Center death rate

Birth year

Apnea

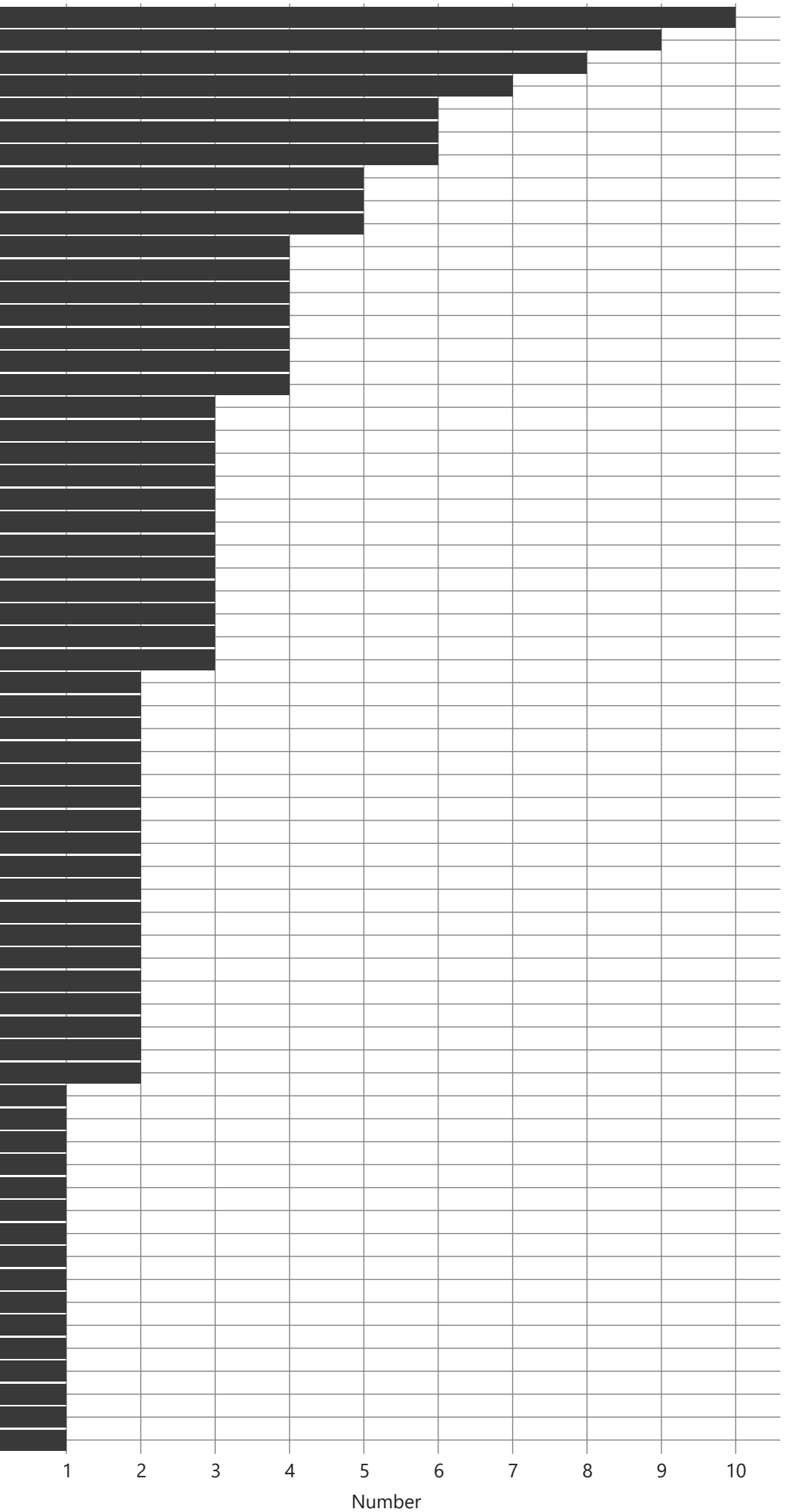

Fig. 4. Frequency of features used by studies. Bar plot displaying features used in included articles according to number of occurrences. 
centage $(27 \%)$ performed external validation. An article by Liu et al. [3] stresses the importance of model validation and reproducibility. In order for a model to achieve universal applicability, it needs to be tested on a dataset outside of its own. Although a model might perform well on its own test data, the true performance of such a model is only known after it is applied to multiple new and unique datasets.

Although this review focused on AI models for mortality, multiple opportunities exist wherein AI can be integrated into the neonatal ICU. In the adult ICU, Awad and colleagues developed a ML model that outperformed highly validated clinical scoring systems [39].In adult medicine, ML prediction models are now integrated into several electronic medical records that flag providers and/or deliver a quantitative risk for developing a future morbidity [40-43]. In a study of 31,000 ICU admissions, Nemati et al. [44] derived an AI prediction model that accurately predicted onset of sepsis up to $12 \mathrm{~h}$ prior to clinical recognition. Another potential area wherein AI can be incorporated in the NICU includes radiograph interpretation. After training an AI algorithm with $>300,000$ chest radiographs, the ability to interpret 72 different findings was comparable to upper level radiology residents [45]. Given that these conditions develop over time, predictive models will improve as more datapoints are fed into the algorithm.

\section{Limitations}

While this study was one of the first of its kind in examining ML and neonatal mortality, it does have some limitations. The first limitation was due to the limited number of articles available on this subject matter and the low quality of methodology reporting in many, this limits the amount of evidence that can be derived. Another issue throughout the articles was the reporting of the details of the dataset used and the ML model development not being consistent throughout the article. The variable nature and inconsistency of reporting in ML studies have been noted to be a problem in previous systematic reviews that examined ML $[12,46]$. Other limitations regard the reporting of the summary measure from the articles, some presented the AUC while others presented the sensitivity and specificity. Without consistent metrics, it limits the ability to compare the studies and models to one another. The authors of this study did not reach out to the authors of the articles included in this study to try and obtain consistent metrics for all the included studies. The last limitation of the study was the inconsistencies with which the included studies reported either a SD or confidence interval for their metrics, with multiple articles not reporting either.

\section{Conclusion}

In conclusion, machine learning models are capable of predicting neonatal deaths. Variability exists with regard to measurement of the model performance, handling of missing data, and validating tools. Less than half of the studies calibrated their models and less than one-third performed external validation. Future studies should focus on external validation, calibration, and deployment of applications that can be readily accessible to health-care providers.

\section{Statement of Ethics}

This study is exempt from Ethics Committee approval since it is a systematic review and only uses previously published literature and does not have any collected patient data.

\section{Conflict of Interest Statement}

The authors have no conflicts of interest to declare.

\section{Funding Sources}

A.M.: Parker B. Francis. Research reported in this publication was supported by the Eunice Kennedy Shriver National Institute Of Child Health \& Human Development of the National Institutes of Health under Award Number K23HD101701. The content is solely the responsibility of the authors and does not necessarily represent the official views of the National Institutes of Health. Both funding sources had no role in this study.

\section{Author Contributions}

C.M. and S.Z. participated in the study design and carried out the data collection, assembled tables, risk of bias, and manuscript writing. K.T. performed the risk of bias. K.C. carried out the data collection and manuscript writing. Ax.M. carried out the data collection. A.M. participated in the study design, advised and carried out data to be collected, supervised all activities, all statistical computations, and the final manuscript drafting. All the authors provided feedback and approved the final manuscript. 


\section{References}

1 World Health Organization. Newborn deaths [Internet] [cited 2021 Mar 2]. Available from: https: //www.who.int/data/gho/data/themes/ topics/indicator-groups/indicator-group-details/GHO/newborn-deaths

2 Goal 3 [Internet]. Department of Economic and Social Affairs [cited 2021 Mar 2]. Available from: https://sdgs.un.org/goals/ goal3? page $=1$.

3 Liu Y, Chen P-HC, Krause J, Peng L. How to read articles that use machine learning: users' guides to the medical literature. JAMA. 2019 Nov 12;322(18):1806-16.

4 Panesar SS, D'Souza RN, Yeh FC, FernandezMiranda JC. Machine learning versus logistic regression methods for 2-year mortality prognostication in a small, heterogeneous glioma database. World Neurosurg X. 2019 Apr 1;2: 100012.

5 Handelman GS, Kok HK, Chandra RV, Razavi $\mathrm{AH}$, Lee $\mathrm{MJ}$, Asadi $\mathrm{H}$. eDoctor: machine learning and the future of medicine. J Intern Med. 2018;284(6):603-19.

6 Deo RC. Machine Learning in Medicine. Circulation. 2015 Nov 17;132(20):1920-30.

7 Safavi KC, Khaniyev T, Copenhaver M, Seelen M, Zenteno Langle AC, Zanger J, et al. Development and validation of a machine learning model to aid discharge processes for inpatient surgical care. JAMA Netw Open. 2019 Dec 2;2(12):e1917221.

8 Moher D, Liberati A, Tetzlaff J, Altman DG, Group TP. Preferred reporting items for systematic reviews and meta-analyses: the PRISMA statement. PLoS Med. 2009 Jul 21;6(7): e1000097.

9 Zupan J, Åhman E. Neonatal and perinatal mortality: country, regional and global estimates. Geneva: World Health Organization 2006. p. 69.

10 Faber FA, Lindmaa A, von Lilienfeld OA, Armiento R. Machine learning energies of 2 million elpasolite ( A B C 2 D 6 ) crystals. Phys Rev Lett. 2016 Sep 20;117(13):135502.

11 Zhang Y, Ling C. A strategy to apply machine learning to small datasets in materials science. Npj Comput Mater. 2018 May 14;4(1): $1-8$.

12 Fleuren LM, Klausch TLT, Zwager CL, Schoonmade LJ, Guo T, Roggeveen LF, et al. Machine learning for the prediction of sepsis: a systematic review and meta-analysis of diagnostic test accuracy. Intensive Care Med. 2020 Mar;46(3):383-400.

13 Wang W, Kiik M, Peek N, Curcin V, Marshall IJ, Rudd AG, et al. A systematic review of machine learning models for predicting outcomes of stroke with structured data. PLoS One. 2020 Jun 12;15(6):e0234722.

14 Moons KG, de Groot JA, Bouwmeester W, Vergouwe Y, Mallett S, Altman DG, et al. Critical appraisal and data extraction for systematic reviews of prediction modelling studies: the CHARMS checklist. PLoS Med. 2014 Oct 14;11(10):e1001744.
15 Peng Y, Nagata MH. An empirical overview of nonlinearity and overfitting in machine learning using COVID-19 data. Chaos Solitons Fractals. 2020 Oct;139:110055.

16 Crowley RJ, Tan YJ, Ioannidis JPA. Empirical assessment of bias in machine learning diagnostic test accuracy studies. J Am Med Inform Assoc. 2020 Jul 1;27(7):1092-101.

17 Ambalavanan N, Carlo WA. Comparison of the prediction of extremely low birth weight neonatal mortality by regression analysis and by neural networks. Early Hum Dev. 2001 Dec;65(2):123-37.

18 Ambalavanan N, Carlo WA, Bobashev G, Mathias E, Liu B, Poole K, et al. Prediction of death for extremely low birth weight neonates. Pediatrics. 2005 Dec;116(6):1367-73.

19 Frize M, Bariciak E, Gilchrist J. PPADS: Physician-PArent Decision-Support for neonatal intensive care. Stud Health Technol Inform. 2013;192:23-7.

20 Frize M, Gilchrist J, Martirosyan H, Bariciak E. Integration of outcome estimations with a clinical decision support system: application in the neonatal intensive care unit (NICU). 2015 IEEE International Symposium on Medical Measurements and Applications (MeMeA) Proceedings. 2015. p. 175-9.

21 Gilchrist J, Ennett CM, Frize M, Bariciak E. Neonatal mortality prediction using real-time medical measurements. 2011 IEEE International Symposium on Medical Measurements and Applications. 2011. p. 65-70.

22 Liu Y, Traskin M, Lorch SA, George EI, Small D. Ensemble of trees approaches to risk adjustment for evaluating a hospital's performance. Health Care Manag Sci. 2015 Mar; 18(1):58-66.

23 Podda M, Bacciu D, Micheli A, Bellù R, Placidi G, Gagliardi L. A machine learning approach to estimating preterm infants survival: development of the Preterm Infants Survival Assessment (PISA) predictor. Sci Rep. 2018 Sep 13;8(1):13743.

24 Shukla VV, Eggleston B, Ambalavanan N, McClure EM, Mwenechanya M, Chomba E, et al. Predictive modeling for perinatal mortality in resource-limited settings. JAMA Netw Open. 2020 Nov 18;3(11):e2026750.

25 Townsend D, Frize M. Complimentary artificial neural network approaches for prediction of events in the neonatal intensive care unit. 2008 30th Annual International Conference of the IEEE Engineering in Medicine and Biology Society. 2008. p. 4605-8.

26 Zernikow B, Holtmannspoetter K, Michel E, Pielemeier W, Hornschuh F, Westermann A, et al. Artificial neural network for risk assessment in preterm neonates. Arch Dis Child Fetal Neonatal Ed. 1998 Sep;79(2): F129-34.

27 Kefi Z, Aloui K, Saber M New approach based on machine learning for short-term mortality prediction in neonatal intensive care unit. Int J Adv Comput Sci Appl. 2019;10(7).
28 Stoltzfus JC. Logistic regression: a brief primer. Acad Emerg Med. 2011 Oct;18(10):1099-104.

29 Brajer N, Cozzi B, Gao M, Nichols M, Revoir M, Balu S, et al. Prospective and external evaluation of a machine learning model to predict in-hospital mortality of adults at time of admission. JAMA Netw Open. 2020 Feb 7;3(2): e1920733.

30 Manz CR, Chen J, Liu M, Chivers C, Regli SH, Braun J, et al. Validation of a machine learning algorithm to predict 180-day mortality for outpatients with cancer. JAMA Oncol. 2020 Nov 1;6(11):1723.

31 Parikh RB, Manz C, Chivers C, Regli SH, Braun J, Draugelis ME, et al. Machine learning approaches to predict 6-month mortality among patients with cancer. JAMA Netw Open. 2019 Oct 25;2(10):e1915997.

32 Kanwal F, Taylor TJ, Kramer JR, Cao Y, Smith D, Gifford AL, et al. Development, validation, and evaluation of a simple machine learning model to predict cirrhosis mortality. JAMA Netw Open. 2020 Nov 3;3(11):e2023780.

33 Shung D, Simonov M, Gentry M, Au B, Laine L. Machine learning to predict outcomes in patients with acute gastrointestinal bleeding: a systematic review. Dig Dis Sci. 2019 Aug; 64(8):2078-87.

34 Luthra AK, Porter K, Hinton A, Krishna SG 82 A comparison of machine learning and logistic regression performance for the prediction of in-hospital mortality among acute biliary pancreatitis patients. Am J Gastroenterol. 2019 Oct;114(1):S48-9.

35 Steyerberg EW, Mushkudiani N, Perel P, Butcher I, Lu J, McHugh GS, et al. Predicting outcome after traumatic brain injury: development and international validation of prognostic scores based on admission characteristics. PLoS Med. 2008 Aug 5;5(8):e165; discussion e165.

36 Caetano SJ, Sonpavde G, Pond GR. C-statistic: a brief explanation of its construction, interpretation and limitations. Eur J Cancer. 2018 Feb;90:130-2.

37 Steyerberg EW, Harrell FE, Borsboom GJJM Eijkemans R, Vergouwe Y, Dik J, et al. Internal validation of predictive models: efficiency of some procedures for logistic regression analysis. J Clin Epidemiol. 2001;54:774-81.

38 Wietlisbach CM. Cooper's fundamentals of hand therapy: clinical reasoning and treatment guidelines for common diagnoses of the upper extremity. 3rd ed. Maryland Heights, MO: Elsevier; 2019.

39 Awad A, Bader-El-Den M, McNicholas J, Briggs J. Early hospital mortality prediction of intensive care unit patients using an ensemble learning approach. Int J Med Inform. 2017 Dec;108:185-95.

40 Tabak YP, Sun X, Nunez CM, Johannes RS. Using electronic health record data to develop inpatient mortality predictive model: Acute Laboratory Risk of Mortality Score (ALaRMS). J Am Med Inform Assoc. 2014 Jun;21(3):455-63. 
41 Rothman MJ, Rothman SI, Beals J. Development and validation of a continuous measure of patient condition using the Electronic Medical Record. J Biomed Inform. 2013 Oct; 46(5):837-48.

42 Churpek MM, Yuen TC, Park SY, Gibbons R, Edelson DP. Using electronic health record data to develop and validate a prediction model for adverse outcomes in the wards. Crit Care Med. 2014 Apr;42(4):841-8.
43 Ye C, Wang O, Liu M, Zheng L, Xia M, Hao $S$, et al. A real-time early warning system for monitoring inpatient mortality risk: prospective study using electronic medical record data. J Med Internet Res. 2019 Jul 5;21(7): e13719.

44 Nemati S, Holder A, Razmi F, Stanley MD, Clifford GD, Buchman TG. An interpretable machine learning model for accurate prediction of sepsis in the ICU. Crit Care Med. 2018 Apr;46(4):547-53.
45 Wu JT, Wong KCL, Gur Y, Ansari N, Karargyris A, Sharma A, et al. Comparison of chest radiograph interpretations by artificial intelligence algorithm vs radiology residents. JAMA Netw Open. 2020 Oct 1;3(10):e2022779.

46 Liu X, Faes L, Kale AU, Wagner SK, Fu DJ, Bruynseels A, et al. A comparison of deep learning performance against health-care professionals in detecting diseases from medical imaging: a systematic review and meta-analysis. Lancet Digit Health. 2019 Oct;1(6):e271-97. 